\title{
Deutsch als Wissenschaftssprache, where are you? Einige Bemerkungen zu den Artikelüberschriften in der deutschen Onlinepresse
}

\author{
German as the language of science, where are you? \\ A few comments on the titles of articles \\ in the German press online
}

\begin{abstract}
The role of the German language in scientific communication in Germany has long been the subject of much discussion. In the media, including online, you can find a numerous articles on the subject. Titles in the press show that the German public is very concerned about this state of affairs. The headlines already leave no illusions that the German language is displaced in the scientific research by the English language. Are we dealing with the birth of new lingua franca? Based on selected titles I tried to show how far they correspond with the content of the article. Subjected analyses allowed to discover language strategies used in the titles to illustrate the problem.
\end{abstract}

KEYWORDS: German language in the scientific communication, English as a new lingua franca, language strategies, titles, English vs. German in scientific communication.

\section{VORBEMERKUNGEN}

Die Rolle der deutschen Sprache in der wissenschaftlichen Kommunikation ist seit langem in der Diskussion. In der Medienwelt, selbstverständlich auch im Internet, finden sich zahlreiche Beiträge zu diesem Thema, die uns mehr oder weniger alarmierende Bedrohungszenarien vor Augen führen. Allein die Überschriften der meisten Texte lassen vermuten, dass die 
deutsche Öffentlichkeit sehr um den Status ihrer Muttersprache in der Welt der Wissenschaften besorgt ist. Am Beispiel der Überschriften ist im vorliegenden Beitrag zu prüfen, welche sprachlichen Strategien bei der Titelgestaltung eingesetzt wurden. Ein guter Titel fesselt die Aufmerksamkeit, ist kurz und bündig, hat Wirkung auf die Emotionen des Lesers, sollte aber gleichzeitig informativ und klar sein.

Zur Analyse habe ich ein Korpus zusammengestellt, das aus 68 Texten/Überschriften besteht. Das Hauptkriterium bei der Wahl der Texte war das Thema. Die Quelle der Texte waren die Onlineausgaben der deutschen Presse, sowohl der überregionalen als auch regionalen. Außer Acht gelassen wurden die österreichischen und schweizerischen Beiträge. Berichte in den Fachzeitschriften wurden auch nicht behandelt. Diese werden nämlich an eine andere Zielgruppe gerichtet, die über ein anderes Vorwissen über die dargestellten Probleme verfügt und das Thema aus einem anderen Blickwinkel als durchschnittliche Leser betrachtet. Mit berücksichtigt wurden dagegen Texte aus den Webseiten wie www.pressetext.com/de, www. dw.de, www.heisse.de. ${ }^{1}$ Das Korpus umfasst Texte aus den Jahren 19962014, die dem Thema Deutsch als Wissenschaftssprache gehören. Die ersten gefundenen Berichte zur Lage der deutschen Wissenschaftssprache stammen aus 1996, dann erscheinen sie bis 2008 in den deutschen Onlinezeitungen und auf den Webseiten nur gelegentlich. Die meisten Texte wurden in der Zeitspanne 2009-2014 veröffentlicht, wobei das Jahr 2014 in dieser Hinsicht als am ertragreichsten erscheint (12 Texte). Die führende Rolle spielen hier die bekannten und oft meinungsbildenden Zeitungen (online Version) in Deutschland. Die behandelten Artikel schildern die Ausführungen über die Position des Deutschen in der wissenschaftlichen Kommunikation, wie auch die damit verbundenen Probleme, ohne sich auf die Wissenschaftssprache eines konkreten Wissenszweiges einzuschränken. Zuerst möchte ich die in den gesammelten Texten behandelte Sachlage schildern. Danach folgt die Analyse der Textüberschriften, wie auch ein kurzes Fazit.

\section{STAND DER DINGE. ENGLISCH DOMINIERT DIE WISSENSCHAFTEN}

Als die erste Wissenschaftssprache der antiken Welt galt das Griechische, das später in der römischen Zeit gleichberechtigt neben dem Lateinischen fortbestand, um seine anerkannte Position im Mittelalter zugunsten des Lateinischen völlig zu verlieren. Die humanistischen Ideale der AufkläEnde.

${ }^{1}$ Vgl. die Auflistung der Titel samt den Medienorganen und dem Erscheinungsdatum am 
rung brachten eine verstärkte Position von Nationalsprachen mit sich und Latein wurde im 16. Jh. als die alleinige Wissenschaftssprache durch Nationalsprachen abgelöst. Heute beobachten wir weltweit einen Rückzug von Nationalsprachen zugunsten einer gemeinsamen Kommunikations- und Wissenschaftssprache, dem Englischen. Im Zeitalter der Globalisierung und Internationalisierung vieler Bereiche ist die Kommunikation über nationale Grenzen hinweg unabdingbar. Von dieser Tendenz bleibt auch die Wissenschaft nicht ausgeschlossen. Da die Forschung heute meistens in internationalen Gruppen betrieben wird, braucht man ein gemeinsames Verständigungsmedium. Dieser gemeinsame Nenner ist seit langem Englisch. Kann man also heute schon von einer neuen Lingua franca sprechen? Hat das Englische den Status erworben, den im Mittelalter Latein genoss? Die Internationalisierung und Globalisierung im Bereich der Lehre und Forschung verursacht dagegen, dass nicht nur Deutsch, sondern auch andere Sprachen im wissenschaftlichen Kontext allmählich an Bedeutung verlieren. Die Forschung wird dadurch einsprachig, was absehbare Konsequenzen sowohl für die Forschung selbst als auch für die Vermittlung des Wissens hat.

\section{TITELANALYSE}

Die Korpustexte führen ein paar Ursachen für das Vorherrschen des Englischen in der wissenschaftlichen Kommunikation an. Es ist unter anderem die schon erwähnte Notwendigkeit, über die Grenzen hinweg zu kommunizieren. Neben den Vorteilen dieser Entwicklung wird auch auf die Kehrseite hingewiesen, was seine Widerspiegelung auch in den Überschriften findet. Dass es Gründe zur Besorgnis um die Zukunft des Deutschen als Wissenschaftssprache gibt, ist allein schon den Titeln des gesammelten Korpus zu entnehmen.

Die Überschrift eines Textes ist sein erstes exponiertes Element und kann auch als Metatext oder eine Art Metaussage zum Text verstanden werden, denn sie gibt oft Informationen über den nachfolgenden Text und charakterisiert ihn. Für die Zeitungskommunikation ist auch die schnelle Erfassbarkeit wichtig, was Lüger (2003: 218) deutlich macht „Je klarer, je eingängiger eine Mitteilung dem Adressaten erscheint, desto größer ist die Chance, dass sie wahrgenommen und für lesenswert gehalten wird" Dafür ist auch die Überschrift verantwortlich, und kann mit Recht als Textwerbung bezeichnet werden. Sie richtet die Aufmerksamkeit des Rezipienten auf ein bestimmtes Element des Textes, manchmal interpretiert sie ihn, wertet ihn und dadurch ruft eine bestimmte Einstellung des Lesers hervor. In 
gewissem Sinne leitet sie den Textempfang. Sie ist eine Art Brücke zwischen dem Text (dem Autor) und dem Leser. Viele unerfahrene Leser lassen sich vom Titel verführen oder sogar irreführen. Sie richten sich nach ihm bei der Wahl des zu lesenden Textes und empfinden ihn als Signal, das darüber entscheidet, ob der Text als lesenswert oder nicht erscheint. „Die Titel als komprimierte Kurztexte vermitteln objektive Sachverhalte in subjektiv strategischer Form" - so Held (1998: 121) und eben diese Strategien sind Gegenstand der vorliegenden Analyse. Die Überschrift lässt auch die Strategien erkennen, die ein bestimmtes Medienorgan vertritt. Will man primär informieren, oder vielleicht die Meinungsbildung des Rezipienten beeinflussen? Bei der Titelanalyse stütze ich mich u. a. auf den von P. Hellwig (1984) verfassten Aufsatz, in dem er das Thema ausführlich erörtert, wie auch auf den Beitrag von Claudio di Meola (1998). Ich konzentriere mich nur auf einen pragmatischen Kontext, in dem die Titel verwendet werden, und zwar - auf den Titel als die Überschrift vor dem vorliegenden Ko-Text. Zu vermerken ist auch, dass im Sinne von Hellwig der Begriff Ko-Text für den sich unter der Überschrift befindenden Text verwendet wird. Darüber hinaus wird auch die Syntax der Überschriften unter die Lupe genommen.

Analysiert wurden folgende Aspekte einer Überschrift: 1. Welche Elemente des Themas wurden im Titel hervorgehoben? 2. Wie wurde das Problem bezeichnet und welche Konnotationen erweckt es? 3. Auf welche Weise wurde das erzielt, also welche sprachlichen/stilistischen Mittel wurden im Titel verwendet? 4. Welche Funktion des Titels überwiegt (Nominativfunktion - Benennung des Textes, deskriptive Funktion - Information über den Inhalt oder pragmatische Funktion - Persuasion/Expressivität/Appell)?

Der Titel/die Überschrift eines Textes enthält oft das Textthema, das nach Agricola (1979: 67) als Grund- und Hauptgedanke, Basisaussage oder Informationskern des Textes und damit ein logischer Bezugspunkt zu verstehen ist. Die Überschrift vermittelt oft die Grundinformation und ist eine Reduktion auf den thematischen Kern des Ko-Textes, seine thematische Paraphrase. Die Überschrift, in der der Grundgedanke (das Thema) realisiert ist, tritt in Form einer Behauptung auf, lässt sich in eine Behauptung transformieren bzw. aus einer solchen ableiten. Viele im Korpus gesammelte Überschriften, sog. beschreibende Titel, kann man als Ergänzung der Phrasen verstehen: Das Thema des Textes ist.../Der Text behandelt.../Der Text behandelt die Frage.../Der Text ist aufzufassen als...: wie z. B. Deutsch in der Wissenschaft (DW), Wissenschaftssprache im Wandel (MB), Bedeutung des Deutschen als Wissenschaftssprache (WB) u. ä.

Die Überschriften thematisieren folgende Probleme: Bedeutungsverlust des Deutschen in der wissenschaftlichen Kommunikation z. B. Deutsch: Wird 
verdrängt (HMoPo) /verliert an Bedeutung (HNA)/wird zweitklassig (BZ)/hat ausgedient (DW)/verschwindet (BMoPo)/gerät in Vergessenheit (FAZ), oder auch anders: Verlust der deutschen Sprache beklagt (SäZ)/Deutsch ade (dw)/ Marginalisierung der deutschen Sprache (FAZ)/Wissenschaft ohne Deutsch(e) (JF). Andererseits wird auf den unaufhaltsamen Vormarsch des Englischen hingewiesen, z. B. Say it in English (FAZ)/Englisch hat Deutsch verdrängt (HMoPo)/Die Wissenschaft spricht Englisch (BZ)/Englisch erobert die Hochschulen (KstA)/English please! (DZ)/Englischbedarf bei Wissenschaftlern (dw)/ Englisch ist auch außerhalb der Naturwissenschaften im Vormarsch (ptxt). Dieser Vorgang hat aber auch eine Kehrseite: Die Wissenschaftler verwenden meistens nur Basisenglisch. Viele Titel nehmen Bezug auf das Englische, wobei sie auch das Deutsche mit berücksichtigen z. B. Der Professor speaks Englisch (WA). Diese Überschrift ist zweisprachig, und erst die Lektüre des Textes gibt Aufschluss über die Einzelheiten der Sachlage. Das Englische der deutschen Professoren klingt einfach stark Deutsch. Sie drücken mit englischen Worten das auf Deutsch Gedachte aus. Oder ein anderes Beispiel: Sprache der Wissenschaft. Say it in broken Englisch (Sl). Damit ist gemeint, dass das Englische oft in seiner Grundform, sog. Globalesisch², verwendet wird, was in den Titeln auch zum Vorschein kommt: Kampf dem Globalesisch (DT)/an den Unis grassiert "BSE“ (Sl)/(Anm. BSE - bad simple Englisch)/Ein bisschen Englisch reicht nicht (SZ)/Der Professor speaks English (WA). Es wird auch auf die Gefahren solcher Entwicklung hingewiesen und Notwendigkeit diese umzukehren z. B. Es ist falsch, wenn nur Englisch gesprochen wird (DW)/Sprachfreies Denken gibt es nicht (FAZ)/Wissenschaftssprache Englisch. Das ist doch absurd (FAZ)/Englisch als Denkbarriere (FAZ)/Dümmer auf Englisch (FAZ)/Englisch ist höchst umstritten (MZ) ${ }^{3}$. Nicht selten zu treffen sind Titel, die anschaulich darauf hinweisen, dass man um Deutsch in der wissenschaftlichen Kommunikation kämpfen soll, z. B. Deutsch als Wissenschaftssprache fördern? (JF)/Professoren kämpfen um Deutsch (HMoPo)/Für Deutsch als Sprache der Wissenschaft (DT)/Wider den Englisch-Wahn an Universitäten (Fs)/Scientific community soll mehr Deutsch wagen (DZ). Das behandelte Problem wird sehr unterschiedlich bezeichnet, einerseits als: Bedeutungsverlust/Wandel/Verdrängung/Marginalisierung/Verschwinden des Deutschen

${ }^{2}$ Globalesisch ist das Wissenschaftsenglisch, das von Nicht-Muttersprachlern gesprochen wird. Der Berliner Sprachwissenschaftler Jürgen Trabant nennt es verächtlich Globalesisch. Der interessierte Leser findet weitere Informationen zu diesem Thema in: Trabant 2014.

${ }^{3}$ Mehr Information zum Deutsch als Wissenschaftssprache findet sich in: Ammon (1998; 2008); Ehlich (1999; 2000; 2006); Klein (2001); Mocikat (2008), wie auch auf der Homepage der Konferenz Deutsch in den Wissenschaften, die vom 10.-12. November 2011 in n Essen (Goethe Institut, DAAD und Institut für Deutsche Sprache) organisiert wurde: <http://www. goethe.de/lhr/prj/diw/deindex.htm> (abgerufen am 28.09.2014). 
aus der wissenschaftlichen Sphäre, sogar als Aussterben der Sprache, andererseits als Eroberung der Hochschulen durch das Englische/Kampf gegen globalisierte Form des Englischen/Kampf um Deutsch/Förderung des Deutschen.

Die gesammelten Beispiele machen deutlich, dass die Textautoren zu einer breiten Palette sprachlicher/ stilistischer Mittel gegriffen haben. Neben sachlicher Information über den im Text behandelten Sachverhalt: Bedeutung des Deutschen als Wissenschaftssprache (WB)/Wissenschaftssprache im Wandel (MB) haben wir hier auch rhetorische Fragen: Die Wissenschaft spricht Englisch. Wird Deutsch zweitklassig? (BZ), Satzellipsen: Deutsch als Wissenschaftssprache erhalten (DW), einfache Sätze: Man spricht (kein) Deutsch (DT), einfache Sätze mit Ergänzungen: Englisch hat Deutsch als Wissenschaftssprache verdrängt ( $\mathrm{HMoPo})$.

Eine interessante Gruppe bilden auch Metaphern. Es handelt sich hier um Überschriften, die nicht so überschaubar sind und vom Leser einen größeren Rezeptionsaufwand erfordern (vgl. Lüger 2003: 219). Hier erscheint oft die Personifizierung des Deutschen ( $\mathrm{ab}$ und zu auch des Englischen), ihm werden Merkmale zugeschrieben, die nur einem Lebewesen zugeschrieben werden. Es wiederholen sich die Wendungen, die mit Leben/Überleben, Tod, Sterben assoziiert werden, z. B. Tot oder lebendig/Deutsch droht auszusterben (heise)/Deutsch liegt im Sterben (MZ)/Deutsch stirbt aus (DZ, DW)/ Deutsch ist kurz vor Aussterben (ptxt)/Ist Deutsch noch zu retten? (DZ)/Soll Deutsch als Wissenschaftssprache überleben? (DZ) Indem man das Deutsche personifiziert, verleiht man der Sprache die Wichtigkeit eines Lebewesens, eines Menschen, dessen Rettung die höchste Pflicht jedes Menschen ist. Diese Metaphern sind informativ, eindeutig und dienen vor allem der Informationsverdichtung, der Verständlichkeit (vgl. di Meola 1998: 224). Sie veranschaulichen deutlich die Sachlage. Bei den Metaphern wie Wider den Englisch-Wahn an Universitäten, oder An den Unis grassiert "BSE” wird auf die Krankhaftigkeit, Unnatürlichkeit der Situation hingewiesen. Der Wahn, BSE (Rinderwahnsinn einerseits, andererseits bad simple Englisch) sind doch Bezeichnungen für schwere Krankheiten. Die Überschrift gibt hier deutlich zu verstehen, dass der Autor diese Erscheinungen als nicht normal, sogar als eine Art Seuche empfindet. Neben den konventionellen Metaphern kommen auch suggestive Metaphern vor (di Meola 1998: 224). Sie unterscheiden sich von den zuerst genannten dadurch, dass sie einen höheren Kreativitätsgrad aufweisen. Sie gelten eher als Leseanreiz als Informationsverdichtung. Als Beispiel kann man hier die Überschriften anführen: Die gebellte Sprache (FAZ)/Die verkannte Muttersprache (DZ). Beide Beispiele beziehen sich auf die geschichtlichen Ursachen dessen, warum Deutsch so unbeliebt ist, d. h. 
auf die Nazizeit, in der Deutsch nur mit der verhassten Sprache des Aggressors assoziiert wurde.

Sehr interessant sind auch die Zitate, die als referenzielle Intertextualität im Sinne von Fix (2000: 453) aufzufassen sind. Dazu gehören Anspielungen an die Liedertitel, z. B. Deutsch ade in der Wissenschaft (dw) ist als Paraphrasierung des Titels "Winter ade, Scheiden tut weh" von Hoffman von Fallersleben (1835) zu interpretieren, und die Überschrift Aber bitte auf Englisch (ND) ist dagegen als intertextuelle Anknüpfung an das bekannte Lied von dem österreichischen Liedermacher Udo Jürgens "Aber bitte mit Sahne" $z u$ deuten. Nebenbei ist zu erwähnen, dass der Titel Aber bitte mit Sahne sich $\mathrm{zu}$ einem geflügelten Wort entwickelte, dass allgemein für einen Zusatz, eine Extraportion o. Ä. verwendet wird. Ähnliches ist über den Titel Kampf dem Globalesisch auszusagen, der auf eine Kampagne Kampf dem Atomtod zurückzuführen sei, die eine außerparlamentarische Widerstandsbewegung in Westdeutschland gegen die Ausrüstung der Bundeswehr mit Atomwaffen und deren Stationierung auf deutschem Boden war. Die Intertextualität kommt auch in einer anderen Art der Bezüge zum Vorschein, nämlich als Anspielungen an die Textmuster (typologische Intertextualität, vgl. Fix 2000: 453). Es gibt z. B. Titel, die mit ihrer Form auf die Märchentitel zurückzuführen sind: Wo Deutsch eine Weltsprache geblieben ist (FAZ)/Wo Forscher noch Deutsch sprechen (DW)/Warum Deutsch als Wissenschaftssprache ausstirbt? (DZ) und zum Vergleich das Märchen Was die Distel erlebte von Hans Christian Andersen, oder Wie der Teufel auf der Flöte blies von Johann Wilhelm Wolf. Die Anspielung an das Textmuster des Märchens ist hier unverkennbar.

Bezüglich der Funktion ist es zu vermerken, dass die Überschriften primär über den Stand der Dinge informieren. Die Autoren der Korpustexte ließen aber den Leser auch mit schauererregenden Titeln überraschen, wie z. B. Tot oder lebendig (heise), Wissenschaftssprache Deutsch droht auszusterben (BMoPo/DW), Wissenschaftssprache Deutsch liegt im Sterben (MZ). Sie sprechen die Emotionen der Rezipienten an und sind Ausdruck von Katastrophismus, Emotionalität, Besorgtheit (expressive Funktion). Manche sind auch als Appell zu deuten: Lernt Englisch! (DW), Forscher mahnen: Schreibt mehr Deutsch (HA). Die zuletzt genannten Beispiele liefern Belege für die persuasive Funktion einer Überschrift.

Die Überschriften der gesammelten Texte reichen von rein informativen, sachlichen Titeln (syntaktisch-semantische Transparenz) z. B. Pro und Contra. Soll Deutsch als Wissenschaftssprache überleben? (DZ)/Deutsch als Wissenschaftssprache erhalten (DW) bis zu primär anregenden, verschlüsselten Titeln (gekennzeichnet durch maximale syntaktisch-semantische Opazität) z. B. Die sprachlose Forschung (DT)/Das Deutsche bringt Klarheit (DT) (vgl. di Meola 
1998: 217). Die ersten informieren einfach über den Inhalt des Ko-Textes, sind leicht interpretierbar, eventuell ist das Implizite problemlos rekonstruierbar (z. B. aus der grammatischen Konstruktion). Sie dienen der Vorinformation. Die Titel der zweiten Gruppe sind oft eine Art Interpretationsspiel, das mit dem Leser betrieben wird. Sie sind häufig rätselhaft formuliert Die verkannte Muttersprache (DZ)/Die gebellte Muttersprache (FAZ), enthalten auch nicht selten formale oder inhaltliche intertextuelle Anspielungen an schon existierende Texte oder Titel (vgl. Fix 2000), indem sie die Phrasen (oder Titel) aus den früher $\mathrm{zu}$ diesem Thema veröffentlichten Texten wiederholen (z. B. von Wissenschaftlern, Politikern oder Experten). Die Überschrift Es ist falsch, wenn nur Englisch gesprochen wird (DW) - führt beispielsweise einen im 2012 durchgeführten Interview mit Horst Hippler dem Chef der Hochschulrektoren geäußerten Satz an. Ähnliche Beispiele liefern die schon früher erwähnten Anspielungen an Liedtexte u. ä. Derartige Überschriften dienen vor allem dem Leseanreiz, spiegeln aber oft die Einstellung oder Wertung des Autors wieder, wie z. B. An den Unis grassiert "BSE”. Oft aber können sie erst zusammen mit dem nachfolgenden Text erschlossen werden.

Nur wenige Titel sind zweiteilig, d. h. bestehen aus einem exponierten Haupttitel und einem Untertitel der voran- oder nachgestellt ist, als Beispiel ist hier folgender Titel anzuführen: Englisch setzt sich als Forschungssprache nicht nur in Natur- sondern auch in Geisteswissenschaften durch. Deutsch verliert an Uni an Bedeutung (HNA). Oft verrät aber erst der Untertitel, wovon der Text handelt, z. B. Tot oder lebendig? Deutsch als Wissenschaftssprache verliert an Bedeutung (heise). Manchmal haben wir es auch mit den Überschriften über einzelnen Textabschnitten zu tun. Diese Aufteilung erleichtert dem Leser die Orientierung im Text, sie ist auch als Hilfe bei der Entschlüsselung des Themas zu verstehen. Von den Korpustexten wurden nur wenige Texte mit Zwischentiteln versehen, und nämlich diese, die einen größeren Umfang aufweisen.

Die im Korpus zusammengestellten Texte gehen über eine reine Nachricht hinaus und als Stellungnahme des Autors zum behandelten Sachverhalt (hier: Deutsch als Wissenschaftssprache) tendieren in Richtung theoretischer Texte (vgl. Hellwig 1984: 11). Der Leser erwartet von solchen Texten die Erklärung und Bewertung der Fakten (Hellwig 1984: 11). Man findet sie meistens in der Rubrik: Feuilleton, Kultur, Wissenschaft.

Das analysierte Korpus enthält verschiedene Typen von Überschriften, sowohl die für die Nachrichtentexte typischen Schlagzeilen z. B. Deutsch als Wissenschaftssprache erhalten (DW)/Deutsch ade in der Wissenschaft (dw) als auch beschreibende Überschriften z. B. Englisch hat Deutsch als Wissenschafts- 
sprache verdrängt (HMoPo), wie auch indirekt beschreibende Überschriften z. B. Der Professors speaks English (...) (Hellwig 1984: 11). Da die Korpustexte als Kommentare zu klassifizieren sind, überwiegt der zweit genannte Typ der Überschrift, und zwar der Titel als Beschreibung des Ko-Textes. Es ist nämlich wichtig, dass der Leser die Texte in der großen Textmenge überhaupt findet, deshalb haben wir es oft mit einer expliziten Angabe des Themas zu tun. Diese Titel informieren noch vor dem Lesen über den Inhalt des KoTextes. Ihre Rolle ist es, das Interesse für den Text zu wecken. Das Thema spiegelt sich oft im Titel in Form einer Frage wieder, zu der es im Ko-Text eine Antwort gibt z. B. Warum eine Wissenschaftssprache Deutsch? (DT)/Ist Deutsch noch zu retten? (DZ)/Wo Forscher noch Deutsch sprechen? (DW).

Obwohl die Schlagzeilen eher für die Nachrichtentexte geeignet sind, sind sie auch hier präsent. Das ist wohl damit zu erklären, dass die in den Texten aufgestellte These - Deutsch verliert in der Wissenschaft an Bedeutung zugunsten des Englischen - schon längst unter Debatte steht und der breiten Öffentlichkeit gut bekannt ist. Der Titel kann also das Vorwissen der Leser ansprechen und muss nicht unbedingt stark informativ sein.

Auch die indirekt charakterisierenden Titel kommen im Korpus vor (obwohl viel seltener); sie erfüllen die Rolle eines Leseanreizes und sollen den Rezipienten neugierig machen. Nur ein Teil derartiger Titel ist dermaßen informativ, dass die Überschrift problemlos ohne Vorwissen erkennen lässt, wovon der Ko-Text handelt. Möglicherweise wurde das Thema in Deutschland bereits so heftig diskutiert, dass es auch dem breiten Publikum bekannt ist, so bedarf es keiner ausführlichen Erklärung. Bei diesem Typ gibt es jedoch manchmal einen Untertitel vom beschreibenden Typ, der den Sachverhalt aufdeckt oder auch einen Vorspann, der Informationsentnahme für den Leser erleichtert und das Verstehen des Textinhalts unterstützt.

Syntaktisch gesehen überwiegen hier die vollständigen Sätze, wobei nicht alle sofort erkennen lassen, was der Artikel zum Thema hat, um hier nur ein paar zu nennen: Say it in English, please (FAZ)/Man spricht (kein) Deutsch (DT)/Das Deutsche bringt Klarheit (DT). Obwohl in den angeführten Titeln kein Element fehlt, erscheinen sie nicht als eindeutig. Sie sind erst nach der Lektüre des nachfolgenden Textes verständlich oder man muss über einen mindestens minimalen Informationshorizont zum jeweiligen Thema verfügen, um sie zu decodieren.

Bei den Überschriften in Form einer syntaktischen Ellipse haben wir auch solche, die trotz der Einsparung eines Elements nichts an der Eindeutigkeit eingebüßt haben, denn die fehlenden Elemente lassen sich oft problemlos aus der grammatischen Konstruktion oder aus dem Zusammenhang erahnen (gänzlich rekonstruierbare Ellipsen). Sie dienen hauptsächlich der 
selektiven Informationsverdichtung (vgl. di Meola 1998: 219), z. B. Deutsch als Wissenschaftssprache erhalten (DW)/Verlust der deutschen Sprache in der Wissenschaft beklagt (SäZ). Bei manchen Überschriften haben wir es mit nicht rekonstruierbaren Ellipsen zu tun, wobei das Implizite nicht aus dem gegebenen Wortmaterial und auch nicht aus dem Vorwissen des Rezipienten rekonstruierbar ist, z. B. Wissenschaftssprache im Wandel (MB). Man weiß nicht, ob hier Deutsch oder eine andere Sprache gemeint ist, unklar bleibt auch, unter welchen Aspekten das Thema behandelt wird und erst die Lektüre des Ko-Textes gibt dem Rezipienten den Aufschluss darüber.

\section{SCHLUSSBEMERKUNGEN}

Dem Korpus ist zu entnehmen, dass dieses Thema seit seiner ersten Nennung immer häufiger präsent ist und immer stärker die Öffentlichkeit bewegt. Das muss bedeuten, dass die Kondition des Deutschen als Wissenschaftssprache sich nicht zum Besseren gekehrt hat. Diese Problematik wird in vielen Medien, von vielen Autoren, wie auch Organisationen und Gesellschaften aufgegriffen. ${ }^{4}$ Wissenschaftler, Publizisten, alle, denen es an der Entwicklung des Deutschen liegt, plädieren für Deutsch als Wissenschaftssprache im Rahmen eines Mehrsprachigkeitskonzepts. Dabei geht es nicht um das Ersetzen des Englischen durch das Deutsche, sondern um eine erneute Etablierung des Deutschen neben dem Englischen. Die Dominanz des Englischen ist in fast allen Kommunikationsbereichen unstrittig, es geht aber um die Sicherung der kulturellen Vielfalt Europas (das macht doch seine Größe aus), wie auch den Erhalt kultureller Identität, die in der Sprache gründet. Die Mehrsprachigkeit bereichert dazu noch die Wissenschaft, indem sie verschiedene Traditionen und Kulturen, wie auch Denkmuster und Argumentationsstrategien einbezieht (vgl. Stickel 2007). ${ }^{5}$ Das behandelte Thema, ist nicht neu, was die Veröffentlichungsdaten der Texte belegen, ist aber in der letzten Zeit immer häufiger in den Medien präsent. Die Zusammenstellung der Überschriften lässt sprachliche Strategien erkennen, die zur Veranschaulichung der Sachlage genutzt wurden. Die Überschriften schildern verschiedene Aspekte des Problems, weisen auf seine Ursachen und Folgen hin. Interessant ist es auch, dass nur wenige Überschriften rein in-

${ }^{4}$ Vgl. u.a. zahlreiche Beiträge in den Publikationen der Gesellschaft für Angewandte Linguistik, z. B. die Bände der Reihe forum für Angewandte Linguistik.

${ }^{5}$ Das Gespräch mit Prof. Dr. Gerhard Stickel, dem Präsidenten der Europäischen Föderation nationaler Sprachinstitutionen, <http://www.goethe.de/lhr/prj/mac/kvi/de1399909.htm> (abgerufen am 28.09.2014). 
formativ sind, die anderen vermitteln neben der Information auch die Emotionen. Ähnlich wie es bei der Konsumwerbung der Fall ist, neigt die Titelgestaltung heute zu Ästhetisierungen und baut auf Überraschungseffekten auf. Held (1998: 122) macht darauf aufmerksam, dass man sich oft der Pointierung, Sensationalisierung und Emotionalisierung bedient, was beim Leser die Lust am Decodieren steigert. Den Titeln ist eine Wertung, Stellungnahme abzulesen. Das ist auch völlig berechtigt, wenn man bedenkt, dass die Autoren selber Deutsch als Muttersprache benutzen. Am Beispiel der Korpustexte ist deutlich sichtbar, dass die Intertextualität hier nur mit Berücksichtigung des Rezipienten betrachtet werden kann, denn von seinem Wissenshorizont hängt $a b$, ob der intertextuelle Bezug der Überschrift enthüllt wird oder nicht (vgl. Fix 2000: 450).

\section{LITERATURVERZEICHNIS}

Agricola, E., 1979. Textstruktur, Textanalyse, Informationskern. Leipzig: Verlag Enzyklopädie.

Ammon, U., 1998 Deutsch oder Englisch?: Über die Wissenschaftssprache der Deutschen. In: Forschung \& Lehre 8/98, 415-417.

Ammon, U., 2008. Deutsch in der internationalen Wissenschaftskommunikation. In: Limbach, J., von Ruckteschell, K. (Hrsg.). Die Macht der Sprache. München: Langenscheidt KG/Goethe-Institut e. V. 47-59.

Ehlich, K., 1999. Alltägliche Wissenschaftssprache. In: Info DaF 26, 1, 3-24.

Ehlich, K., 2000. Deutsch als Wissenschaftssprache für das 21. Jahrhundert. <http:/ / www.gfljournal.de/Issue_1_2000.php> (abgerufen am 26.09.2014).

Ehlich, K., 2006. Mehrsprachigkeit in der Wissenschaftskommunikation - Illusion oder Notwendigkeit? In: Ehlich, K., Heller, D. (Hrsg.). Die Wissenschaft und ihre Sprachen. Bern: Lang, 17-38.

Fix, U., 2000. Aspekte der Intertextualität. In: Brinkner, K., Antos, G., Heinemann, W., Sager S.F. (Hrsg.). Text- und Gesprächslinguistik. Ein internationales Handbuch zeitgenössischer Forschung. 1. Halbband. Berlin-New York: Walter de Gruyter \&Co, 449-457.

Held, G., 1998. Der Titel als Leseerlebnis. Journalistische Spielarten in den Schlagzeilen italienischer Nachrichtenmagazine. In: Kettemann, B., Stegn, M., Stöckl, H. (Hrsg.). Mediendiskurse. Frankfurt am Main: Peter Lang.

Hellwig, P., 1984. Titulus oder über den Zusammenhang von Titeln und Texten. Titel sind ein Schlüssel zur Textkonstitution. In: Henne, H. (Braunschwieg), Oksar, E. (Hamburg), von Polenz, P. (Trier), Wiegand, H.E. (Heidelberg) (Hrsg.). Zeitschrift für germanistische Linguistik, Berlin: Walter de Gruyter \& Co.,1-20.

Klein, W., 2001. Das Ende vor Augen: Deutsch als Wissenschaftssprache. In: Debus, F., Kollmann, F., Pörken, U. (Hrsg.). Deutsch als Wissenschaftssprache im 20. Jahrhundert Mainz: Akademie der Wissenschaften und der Literatur, 289-293.

Lüger, H. (Hrsg.), 2003. Anpassung und Wandel in der elsässischen Regionalpresse. Im Blickpunkt: das ELSASS. Landau: Knecht, 207-230

Meola, C., di, 1998. Zur Syntax von Schlagzeilen in der deutschen Tagespresse. In: Muttersprache Nr. 108, 217-231. 
Mocikat, R., 2008. Die deutsche Sprache in den Naturwissenschaften. In: Limbach, J., von Ruckteschell, K. (Hrsg.). Die Macht der Sprache. München: Langenscheidt KG-GoetheInstitut e. V. 60-65.

Trabant, J., 2014. Globalesisch oder was? Ein Plädoyer für Europas Sprachen. München: C.H. Beck.

<http://www.goethe.de/lhr/prj/diw/deindex.htm> (abgerufen am 28.09.2014).

<http://www.goethe.de/lhr/prj/mac/kvi/de1399909.htm> (abgerufen am 28.09.2014).

\section{DIE BEI DER TITELANALYSE BERÜCKSICHTIGTEN ARTIKEL SAMT QUELLEN UND ERSCHEINUNGSDATUM, WIE AUCH DIE IM TEXT VERWENDETEN ABKÜRZUNGEN DER MEDIENORGANE (ABGERUFEN AM 22.11.2014)}

\section{Die Welt/DW}

1. Deutsch stirbt an der Uni aus (27.03.2001),

http:/ / www.welt.de/ print-welt/article442071/Deutsch-stirbt-an-der-Uni-aus.html

2. Lernt Englisch! (19.01.2002), http://www.welt.de/print-welt/article423481/Lernt-Englisch.html

3. Wo Forscher noch Deutsch sprechen (24.01.2009), http://www.welt.de/welt_print/article3081919/Wo-Forscher-noch-Deutschsprechen.html

4. Wissenschaftssprache Deutsch droht auszusterben (27.01.2009), http://www.welt.de/wissenschaft/article3100367/Wissenschaftssprache-Deutschdroht-auszusterben.html

5. Deutsch als Wissenschaftssprache erhalten (27.01.2009), http:// www.welt.de/debatte/kommentare/article6073843/Deutsch-alsWissenschaftssprache-erhalten.html

6. Deutsch in der Wissenschaft (28.01.2009), http://www.welt.de/welt_print/article3102824/Deutsch-in-der-Wissenschaft.html

7. Warum die deutsche Sprache überleben wird (10.03.2010), http://www.welt.de/debatte/kommentare/article6719247/Warum-die-deutscheSprache-ueberleben-wird.html

8. Hat Deutsch in der Wissenschaft ausgedient? (20.11.2011), http:/ / www.welt.de/print/wams/vermischtes/article13725995/Hat-Deutsch-in-derWissenschaft-ausgedient.html

9. „Es ist falsch, wenn nur Englisch gesprochen wird” (2.05.2012), http:/ / www.welt.de/politik/deutschland/article106252104/Es-ist-falsch-wenn-nurEnglisch-gesprochen-wird.html

10. Warum Deutsch als Forschungssprache verschwindet (27.01.2013), http:/ / www.welt.de/politik/deutschland/article113150770/Warum-Deutsch-alsForschungssprache-verschwindet.html

11. Man spricht nicht mehr Deutsch (9.03.2014), http:// www.welt.de/kultur/article125606862/Man-spricht-nicht-mehr-deutsch.html 


\section{Frankfurter Allgemeine/FAZ}

1. Dümmer auf Englisch? „Die Marginalisierung der deutschen Sprache in der Wissenschaft" (19.11.2007),

http:/ / www.faz.net/aktuell/feuilleton/geisteswissenschaften/wissenschaftsspracheduemmer-auf-englisch-1461421.html

2. Deutsch als Wissenschaftssprache. Sprachfreies Denken gibt es nicht (25.04.2008), http://www.faz.net/aktuell/ feuilleton/deutsch-als-wissenschaftssprachesprachfreies-denken-gibt-es-nicht-1544592.html

3. Wissenschaftssprache Englisch. Das ist doch absurd (25.04.2008), http:/ / www.faz.net/aktuell/beruf-chance/campus/wissenschaftssprache-englischdas-ist-doch-absurd-1538756.html

4. Say it in English, please (30.04.2008), http:// www.faz.net/aktuell/beruf-chance/campus/sprache-und-wissenschaft-say-itin-english-please-1544129.html

5. Wissenschaftlicher Zitatindex. Lasst Euch nicht erpressen! (10.03.2012), http://www.faz.net/aktuell/feuilleton/forschung-und-lehre/wissenschaftlicherzitatindex-lasst-euch-nicht-erpressen-11674256.html

6. Englisch als Denkbarriere (4.08.2014), http://www.faz.net/aktuell/feuilleton/geisteswissenschaften/englisch-alsdenkbarriere-1160133.html

7. Angewandte Chemie. Wo Deutsch eine Weltsprache geblieben ist (4.08.2014), http:/ / www.faz.net/aktuell/wissen/physik-chemie/angewandte-chemie-wodeutsch-eine-weltsprache-geblieben-ist-1489689.html

8. Deutsch. Die gebellte Sprache (4.08.2014), http:/ / www.faz.net/aktuell/feuilleton/geisteswissenschaften/deutsch-die-gebelltesprache-1542420.html

\section{Der Tagesspiegel/DT}

1. Warum eine Wissenschaftssprache Deutsch? (13.02.2002), http:/ / www.tagesspiegel.de/weltspiegel/gesundheit/warum-einewissenschaftssprache-deutsch/290400.html

2. "Deutsch ist eine wunderbare Sprache” (23.01.2004), http://www.tagesspiegel.de/weltspiegel/gesundheit/deutsch-ist-eine-wunderbaresprache/484320.html

3. Für Deutsch als Sprache der Wissenschaft (22.08.2005),

http://www.tagesspiegel.de/weltspiegel/gesundheit/fuer-deutsch-als-sprache-derwissenschaft/635368.html

4. Wie die Deutsche Sprache überleben kann (1.11.2007), http:/ / www.tagesspiegel.de/wissen/wie-die-deutsche-sprache-ueberlebenkann/1083616.html

5. Für mehr Deutsch in der Wissenschaft (11.11.2011), http:/ / www.tagesspiegel.de/wissen/fuer-mehr-deutsch-in-derwissenschaft/5822806.html

6. Das Deutsche bringt Klarheit (20.03.2011), http:/ / www.tagesspiegel.de/wissen/turners-thesen-das-deutsche-bringtklarheit/3969920.html 
7. Die sprachlose Forschung (1.10.2011), http://www.tagesspiegel.de/meinung/diesprachlose-forschung/4679386.html

8. Deutsch als Wissenschaftssprache. Kampf dem Globalesisch (5.12.2012), http:/ / www.tagesspiegel.de/wissen/deutsch-als-wissenschaftssprache-kampf-demglobalesisch/7477240.html

9. Was tun für Deutsch in der Wissenschaft? (25.01.2013), http:/ / www.tagesspiegel.de/wissen/was-tun-fuer-deutsch-in-derwissenschaft/7684238.html

10. Man spricht (kein) Deutsch (23.02.2013), http://www.tagesspiegel.de/zeitung/man-spricht-kein-deutsch/7825486.html

11. Deutsch 3.0 (30.01.2014), http:/ / www.tagesspiegel.de/wissen/themen-und-termine-deutsch-3-0/9410438.html

12. Sprachdebatten mit dem Goethe- Institut. Deutsch, aber witzig (31.01.2014) http://www.tagesspiegel.de/wissen/sprachdebatten-mit-dem-goethe-institutdeutsch-aber-witzig/9410434.html

\section{Die Zeit/DZ}

1. Warum Deutsch als Wissenschaftssprache ausstirbt? (19.07.1996), http://www.zeit.de/1996/30/deutsch.txt.19960719.xml

2. Die verkannte Muttersprache (6.09.1996), http://www.zeit.de/1996/37/Die_verkannte_Muttersprache

3. Anglais oblige? (26.07.2007), http:/ / www.zeit.de/2007/31/Deutsch-Brain-up

4. Deutsche Sprache: Ist Deutsch noch zu retten? (8.07.2010) http:/ / www.zeit.de/2010/27/Deutsch-Muttersprache

5. Pro und contra. Soll Deutsch als Wissenschaftssprache überleben? (25.01.2011), http://www.zeit.de/wissen/2010-04/deutsch-forschungssprache

6. English please! (2.08.2012), http:/ / www.zeit.de/2012/32/C-Wissenschaftssprache

7. Scientific community soll mehr Deutsch wagen (5.12.2012), http://www.zeit.de/wissen/2012-12/Wissenschaftssprache-Deutsch

\section{Süddeutsche Zeitung/SZ}

1. Ein bisschen Englisch reicht nicht (11.11.2010), http:// www.sueddeutsche.de/karriere/wissenschaftliche-aufsaetze-ein-bisschenenglisch-reicht-nicht-1.986666

2. Streit in der Wissenschaft. Wer Leser will, muss auf Englisch schreiben (23.07.2014) http:/ / www.sueddeutsche.de/bildung/streit-in-der-wissenschaft-pflichtspracheenglisch-1.2018594

3. In English please! (23.07.2014), http:/ / www.sueddeutsche.de/muenchen/tu-muenchen-in-english-please-1.2058535

\section{Mitteldeutsche Zeitung/MZ}

1. Professoren kämpfen um Deutsch (26.07.2001), http:/ / www.mz-web.de/kultur/wissenschaftssprache-professoren-kaempfen-umdeutsch,20642198,20028628.html 
2. Wissenschaftssprache Deutsch liegt im Sterben (27.01.2009), http:/ / www.mz-web.de/campus/-wissenschaftssprache-deutsch-liegt-im-sterben,20641608,18206512.html

3. Englisch ist höchst umstritten (17.11.2009), http:/ / www.mz-web.de/ koethen/englisch-ist-hoechstumstritten,20641024,17932674.html

\section{Berliner Morgenpost/BMoPo}

1. Wissenschaftssprache Deutsch droht auszusterben (27.01.2009), http://www.morgenpost.de/web-wissen/article1023020/WissenschaftsspracheDeutsch-droht-auszusterben.html

\section{Hamburger Morgenpost/HMoPo}

1. Englisch hat Deutsch als Wissenschaftssprache verdrängt (18.01.2000), http:/ / www.mopo.de/news/dienstag-18-01-2000-06-11-englisch-hat-deutsch-alswissenschaftssprache-verdraengt,5066732,6296116.html

2. Professoren kämpfen um Deutsch als Wissenschaftssprache (26.07.2001), (dpa) http:/ / www.mopo.de/news/donnerstag-26-07-2001--12-18-professoren-kaempfenum-deutsch-als-wissenschaftssprache,5066732,6028648.html

\section{Badische Zeitung/BZ}

1. Die Wissenschaft spricht Englisch: Wird Deutsch zweitklassig? (11.09.2010), http:/ / www.badische-zeitung.de/kommentare-1/die-wissenschaft-spricht-englischwird-deutsch-zweitklassig--35272299.html

\section{KöIner Stadtanzeiger/KstA}

1. Englisch erobert die Hochschulen (11.06.2007), http://www.ksta.de/campus/englisch-erobert-diehochschulen,15189650,13423128.html

\section{Hessische niedersächsische Allgemeine/HNA}

1. Deutsch verliert an Uni an Bedeutung (2.02.2001), http:// www.hna.de/kassel/stadt-kassel/deutsch-verliert-bedeutung-3345842.html

\section{Neues Deutschland/ND}

1. Aber bitte auf Englisch (29.07.2011), https://www.neues-deutschland.de/artikel/203174.aber-bitte-auf-englisch.html

\section{Hamburger Abendblatt/HA}

1. Forscher mahnen: Schreibt mehr Deutsch (7.06.2012), http:/ / www.abendblatt.de/ratgeber/wissen/article2299688/Forscher-mahnenSchreibt-mehr-Deutsch.html

\section{Westdeutsche Allgemeine/WA}

1. Der Professor speaks English (10.02.2009), http:/ / www.derwesten.de/wr-info/der-professor-speaks-english-id283236.html 


\section{Mittelbayerische/MB}

1. Wissenschaftssprache im Wandel (4.07.2014)

http://www.mittelbayerische.de/nachrichten/wissenschaft/wissenschaft/artikel/wi ssenschaftssprache-im-wandel/1088223/wissenschaftssprache-im-wandel.html

\section{Wochenblatt/WB}

1. Bedeutung des Deutschen als Wissenschaftssprache (27.06.2014), http://www.wochenblatt.de/bildung/regensburg/uni-regensburg/Bedeutung-desDeutschen-als-Wissenschaftssprache;art12383,250288

\section{Sächsische Zeitung/SäZ}

1. Verlust der deutschen Sprache in der Wissenschaft beklagt (27.01.2013), http:/ / www.sz-online.de/nachrichten/wissen/verlust-der-deutschen-sprache-in-derwissenschaft-beklagt-2494612.html

\section{Focus online/Fs}

1. Wider den Englisch- Wahn an Universitäten (21.11.2013), http://www.focus.de/politik/experten/rehlaender/not-amused-wider-den-englischwahn-an-universitaeten_id_3419991.html

\section{Der Spiegel online/SI}

1. An den Unis grassiert „BSE” (17.01.2002), http://www.spiegel.de/unispiegel/wunderbar/sprachwaechter-an-den-unisgrassiert-bse-a-177617.html

2. Sprache der Wissenschaft: Say it in broken Englisch (3.10.2003), http:// www.spiegel.de/unispiegel/studium/sprache-der-wissenschaft-say-it-inbroken-english-a-440120.html

\section{Junge Freiheit)/JF}

1. Deutsch als Wissenschaftssprache fördern? (3.10.2003), http://www.jf-archiv.de/archiv03/413yy07.htm

2. "Nicht ohne Not verzichten" (10.10.2003), http://www.jf-archiv.de/archiv03/423yy09.htm

3. Wissenschaft ohne Deutsch(e) (26.07.2014), http://jungefreiheit.de/kolumne/2014/wissenschaft-ohne-deutsche/

\section{www.heise.de/heise}

1. Tot oder lebendig? Deutsch als Wissenschaftssprache verliert an Bedeutung (24.05.2010), http://www.heise.de/tp/artikel/32/32509/1.html

\section{www.dw.de/dw}

1. Englischbedarf bei Wissenschaftlern (15.03.2011), ww.dw.de/englischbedarf-bei-wissenschaftlern/a-6471123

2. Deutsch ade in der Wissenschaft? (8.11.2011), http://www.dw.de/deutsch-ade-in-der-wissenschaft/a-15517302

3. Wissenschaftssprache Deutsch nicht ersetzbar (14.11.2011), http:/ / www.dw.de/wissenschaftssprache-deutsch-nicht-ersetzbar/a-6658885 
4. Man liest nicht länger Deutsch http://dw.de/p/KyNB

\section{www.pressetext.com/ptxt}

1. Deutsch in Wissenschaft kurz vor Aussterben. Englisch auch außerhalb der Naturwissenschaften im Vormarsch (14.10.2009),

http://www.pressetext.com/news/20091014047 
